\title{
Long term outcomes in diabetic patients treated with atherectomy for peripheral artery disease
}

\author{
Adam J. Janas ${ }^{1,2}$, Krzysztof P. Milewski ${ }^{1}$, Piotr P. Buszman ${ }^{1,3}$, Wojciech Trendel ${ }^{1,2}$, \\ Aleksandra Kolarczyk-Haczyk ${ }^{1,2}$, Mariusz Hochuł ${ }^{1}$, Maciej Pruski Jr. ${ }^{1}$, \\ Wojciech Wojakowski ${ }^{1,4}$, Pawel E. Buszman ${ }^{1}$, Radosław S. Kiesz ${ }^{1,2}$ \\ ${ }^{1}$ Center of Cardiovascular Research and Development by American Heart of Poland, Katowice, Poland \\ ${ }^{2}$ San Antonio Endovascular and Heart Institute, Texas, United States \\ ${ }^{3} 3^{\text {rd }}$ Department of Cardiology, Silesian Center for Heart Disease, Katowice, Poland \\ ${ }^{4} 3^{\text {rd }}$ Department of Cardiology, Clinical Hospital of the Silesian Medical University, Katowice, Poland
}

\begin{abstract}
Background: The prevalence of diabetes has increased significantly in well-developed countries during the last decade and it continues to grow. Diabetes increases the risk of restenosis in patients treated percutaneously for peripheral artery disease. The present study sought to compare outcomes of atherectomy treatment in diabetic (DM) vs. non-diabetic (nDM) patients suffering from peripheral artery disease.

Method: Between 2008 and 2012, 204 revascularization atherectomy procedures were performed on arteries of the lower extremities. The endpoints included target lesion revascularization (TLR), amputation and death. The type of atherectomy (excisional-soft plaque, orbital-calcified plaque, with active aspiration - with a thrombus) was left to operator discretion.

Results: This study contains 132 DM (66\% male, age $68 \pm 11.2$ years) and $72 \mathrm{nDM}$ (63\% male, age $75 \pm 11.3$ years) subjects. DM were younger but had a higher prevalence of coronary artery disease (DM: $91 \%$ vs. $n D M: 62 \%, p<0.0001$ ) and end-stage renal disease (DM: $22 \%$ vs. $n D M: 2.5 \%, p<0.0001$ ). There were no differences in critical limb ischemia between the groups (DM: $21 \%$ vs. $n D M: 12 \%, p=$ $=0.13)$. Mean time of follow-up was 384 and 411 days in DM and $n D M$, respectively $(p=0.43)$. There were no significant differences in TLR (DM: $15.2 \%$ vs. $n D M: 22.2 \%, p=0.249$ ), amputations (DM: $3.0 \%$ vs. $n D M: 1.5 \%, p=N S$ ) or death rates (DM: $2.2 \%$ vs. $n D M: 2.7 \%, p=N S)$. Kaplan-Mayer analysis showed no significant differences between the groups in the time to TLR, amputation or death. Conclusions: Plaque modification with adjusted atherectomy appears to have similar outcomes in diabetic as well as in non-diabetic patients. Nonetheless, a randomized study would be warranted to confirm the findings of the current study. (Cardiol J 2020; 27, 5: 600-607)
\end{abstract}

Key words: atherectomy, diabetes mellitus, peripheral artery disease, critical limb ischemia, claudication, below the knee, above the knee

\section{Introduction}

Diabetes mellitus (DM) has a pandemic status in well-developed countries. It is projected that DM will have a prevalence of 552 million worldwide by 2030 [1]. The strongest risk factors for peripheral artery disease (PAD) are DM and smoking [2].
Whereas the ratio of smokers is falling, the DM prevalence continues to increase. The symptomatic PAD is observed in $21 \%$ of patients with DM [3]. Moreover, DM is also an independent risk factor for chronic kidney disease which significantly increases the chance of PAD [4]. Over the years multiple therapies for PAD have emerged, includ-

Address for correspondence: Adam Janas, MD, PhD, Center of Cardiovascular Research and Development by American Heart of Poland, ul. Czajek 41, 40-534 Katowice, Poland, tel: +48602332935, e-mail: adamjjanas@gmail.com 
ing pharmacological regimens, endovascular and open surgery, drug-coated balloons, and stem cell therapy [5]. Nevertheless, revascularization of lower limb arteries in patients with DM brings disappointing long-term outcomes in comparison to the non-diabetic population $[6,7]$. This could be caused by the fact that diabetic lesions in diabetic patients occur over a wider area of the vasculature, including small-diameter vessels [8]. As a result, the atherectomy type chosen based on the plaque morphology and vessel diameter may improve long-term outcomes [9].

The long-term outcomes of endovascular revascularization of lower limb arteries using atherectomy in diabetic patients remains unclear. Therefore, the aim of this study to is compare long-term outcomes after endovascular revascularization of lower limb arteries with atherectomy in diabetic (DM) and non-diabetic (nDM) patients.

\section{Methods}

\section{Subjects}

This study is based on a retrospective study of 203 consecutive patients with symptomatic PAD who underwent endovascular revascularization with atherectomy between 2008 and 2012 at San Antonio Endovascular and Heart Institute. 132 patients were diabetic, whereas 72 were non-diabetic.

Adult patients ( $>18$ years old) with both intermittent claudication (Rutherford 3) and critical limb ischemia (CLI; Rutherford 4-6) were included provided they had at least 1 lesion with $>70 \%$ diameter stenosis confirmed on live quantitative vessel angiography in a lower extremity artery. Patients with in-stent restenosis and diabetes type 1 were excluded.

\section{Procedural characteristic and pharmacological regimen}

Directional (Silver Hawk ${ }^{\mathrm{TM}}$, Medtronic), orbital (Diamondback $360^{\circ}, \mathrm{CSI} 360^{\circ}$ ) and directional with suction (Jetstream ${ }^{\mathrm{TM}}$, Boston Scientific) atherectomy (AT) devices were applied in this study. The type of AT was left to operator discretion, nonetheless directional AT was performed in soft and mixed plaques; orbital AT was applied when a lesion appeared to be calcified; and directional AT with suction was performed when thrombus was suspected. Orbital AT was always followed by the low-pressure balloon post-dilatation; and after directional AT, percutaneous transluminal angioplasty (PTA) was performed if residual stenosis was $>30 \%$. The distal protection system was not used for any patient. Angiographic success was defined as post-procedural Thrombolysis in Myocardial Infarction (TIMI) 3 flow, no dissection or residual stenosis $<30 \%$. If angiographic success was not achieved, bail-out stenting was performed. Acetylsalicylic acid ( $81 \mathrm{mg} /$ day) was continued indefinitely whereas clopidogrel $(75 \mathrm{mg} /$ /day) was advised to be continued for 12 months after the procedure together with atorvastatin, at the maximum tolerable dose, usually $40 \mathrm{mg}$ daily.

\section{Atherectomy devices}

The Silver Hawk plaque excision system (Medtronic) is a forward cutting directional AT device. The device consists of a rotating blade inside a tubular housing with a collection space in the nose cone. The device enables the performance of AT in vessels with a diameter of $1.5-7 \mathrm{~mm}$.

Diamondback $360^{\circ}\left(\mathrm{CSI} 360^{\circ}\right)$ is an orbital AT system tipped with an eccentric, diamond-coated crown. The crown rpm can vary from 60,000 to 200,000 . The crown may be advanced forward and backward when it is intra-arterial. The needed diameter is achieved by increasing the speed of rotation. Faster speeds result in an increased centrifugal force, yielding a larger orbit, and this device is recommended for calcified lesions. Usually, orbital AT is performed before stenting/balloon angioplasty.

The Pathway Jetstream PV Atherectomy System (Boston Scientific) is a rotational AT device with a front-cutting tip that spins at 60-70,000 rpm. Jetstream $^{\circledR}$ expandable catheters have a catheter tip that remains at a diameter of 2.1-2.4 $\mathrm{mm}$ when rotating clockwise and $2.4-3.4 \mathrm{~mm}$ when rotating counterclockwise. For below the knee interventions this device is available in a fixed size: $1.6 \mathrm{~mm}$ and $1.85 \mathrm{~mm}$. This is the only AT device on the market with active aspiration. The derbies as well as thrombus are collected in a bag located on the console device, outside the body.

\section{Study endpoints and definitions}

Because of the observational nature of this study, no preliminary hypothesis was generated. Target lesion revascularization (TLR) was considered a primary endpoint and was defined as any symptom-driven revascularization within a previously treated segment. Unplanned amputation related to a previously treated vessel, death and a change in the Rutherford class were regarded as secondary endpoints. Furthermore, incidents of vessel perforation, dissection and distal embolization, and bailout stenting were collected. 
Table 1. Demographics.

\begin{tabular}{lccc}
\hline & Non-diabetic patients & Diabetic patients & P \\
\hline Number & 72 & 132 & 0.7 \\
Male & $46(63 \%)$ & $88(66 \%)$ & 0.0001 \\
Age $[$ years] & $75 \pm 11.3$ & $68 \pm 11.2$ & $<0.0001$ \\
Body mass index $\left[\mathrm{m} / \mathrm{kg}^{2}\right]$ & $26.5 \pm 4.9$ & $29.4 \pm 4.8$ & $<0.0001$ \\
Coronary artery bypass grafting & $10(14 \%)$ & $56(42 \%)$ & 0.0185 \\
Percutaneous coronary intervention & $30(41 \%)$ & $79(60 \%)$ & 0.163 \\
Previously revascularized peripheral artery disease & $1(5.5 \%)$ & $8(6.0 \%)$ & 0.9 \\
Arterial hypertension & $71(99 \%)$ & $132(100 \%)$ & $<0.0001$ \\
Coronary artery disease & $45(62 \%)$ & $119(91 \%)$ & 0.1332 \\
Critical limb ischemia & $9(12 \%)$ & $28(21 \%)$ & $<0.0001$ \\
Dialysis reliant & $2(2.5 \%)$ & $42(22 \%)$ & 0.8445 \\
Smokers & $11(15 \%)$ & $23(17.4 \%)$ & \\
\hline
\end{tabular}

\section{Safety and ethics}

This retrospective study was conducted in accordance with standard ethics guidelines. Endovascular procedures were carried out by experienced interventional cardiovascular teams in a high-volume center with a vascular surgery back up within 30 min of transportation.

Owing to the observational and retrospective nature of this study, neither patient consent nor ethics committee approval was required.

\section{Data collection and follow-up}

Clinical and procedural data were collected on case report forms generated by the hospital electronic system, containing all patient hospitalization and discharge information. This system is audited for institutional quality assurance by private insurance companies and the state health fund.

Long-term follow-up data were collected during ambulatory check-ups or over the phone. The follow-up office visits were usually scheduled every 3-5 months. Some patients had phone consultations due to a lack of symptoms, and office-based follow-ups were scheduled on a further date. All outcomes of interest were confirmed using hospital discharge charts. Three patients met exclusion criteria for in-stent restenosis and 3 were lost to follow-up.

\section{Statistical analysis}

Continuous variables are presented as mean \pm standard deviation or median (interquartile range $[\mathrm{IQR}]$ ). Data were compared using the t-test for parametric or Mann-Whitney U-test for nonparametric continuous variables. Categorical vari- ables are reported as frequencies (percentages) and were compared using the $\chi^{2}$ or Fisher exact test, as appropriate. Survival curves were constructed using the Kaplan-Meier estimates and were compared with the log-rank test. All reported p-values are two-tailed, and $\mathrm{p}<0.05$ was considered as significant. GraphPad 6 Prism was used for statistical analysis.

\section{Results}

The method of diabetes control was primarily oral agents $(56.3 \%, \mathrm{n}=73)$ followed by insulin injections $(36.6 \%, \mathrm{n}=48)$ or a combination of both $(8.3 \%, \mathrm{n}=11)$. Patients in the diabetic cohort were significantly younger but had considerably more risk factors including off-range body mass index, coronary artery disease, coronary artery bypass grafting, percutaneous coronary interventions and dialysis-dependent renal insufficiency (Table 1). The mean time of follow-up was 384 and 411 days in $\mathrm{DM}$ and $\mathrm{nDM}$, respectively $(\mathrm{p}=0.43)$.

Lesion characteristics were similar in DM $(\mathrm{n}=198)$ and $\mathrm{nDM}(\mathrm{n}=106)$ patients with a mean number of lesions per patient equaling 1.5 in both groups. Lesion location was primarily superficial femoral artery in $\mathrm{nDM}(33 \%, \mathrm{n}=38)$, whereas in the DM cohort anterior tibial artery was most frequently revascularized $(29.7 \%, \mathrm{n}=59)$. There were no significant differences in target lesions between the groups. Furthermore, there were no differences between the groups in terms of lesion morphology in the TransAtlantic Inter-Society Consensus (TASC) and the number of total chronic occlusion. There were no significant differences 
Table 2. Procedural characteristics.

\begin{tabular}{|c|c|c|c|}
\hline & Non-diabetic patients & Diabetic patients & $\mathbf{P}$ \\
\hline Number & 106 & 198 & \\
\hline Iliac & 0 & $1(0.5 \%)$ & 1.0 \\
\hline Common femoral artery & $1(0.94 \%)$ & $1(0.5 \%)$ & 1.0 \\
\hline Superficial femoral artery & $38(35.8 \%)$ & $41(20.7 \%)$ & 0.064 \\
\hline Profunda femoral artery & $0(0 \%)$ & $1(0.05 \%)$ & 1.0 \\
\hline Popliteal artery & $9(8.4 \%)$ & $23(11.6 \%)$ & 0.09 \\
\hline Anterior tibial artery & $20(18.8 \%)$ & $59(29.7 \%)$ & 0.265 \\
\hline Trunk & $5(4.9 \%)$ & $13(6.5 \%)$ & 0.471 \\
\hline Peroneal artery & $10(9.4 \%)$ & $18(9 \%)$ & 0.51 \\
\hline Dorsalis pedis & $3(2.8 \%)$ & $5(2.5 \%)$ & 0.173 \\
\hline Calcaneal artery & $3(2.8 \%)$ & $5(2.5 \%)$ & 0.173 \\
\hline Above the knee & 49 & 67 & \\
\hline Below the knee & 57 & 131 & \\
\hline Graft & 1 & 8 & 0.086 \\
\hline Pre-procedure (\% diameter stenosis) & $89.7 \%$ & $93.7 \%$ & 0.386 \\
\hline Mean lesion length [mm] & $76 \pm 23$ & $81 \pm 19$ & 0.148 \\
\hline TASC A & $18(36.7 \%)$ & $24(35.8 \%)$ & 1.0 \\
\hline TASC B & $15(30.6 \%)$ & $18(26.9 \%)$ & 0.681 \\
\hline TASC C & $11(22.5 \%)$ & $17(25.4 \%)$ & 0.827 \\
\hline TASC D & $5(10.2 \%)$ & $8(11.9 \%)$ & 1.00 \\
\hline Chronic total occlusion & $20(18.8 \%)$ & $56(28.2 \%)$ & 0.073 \\
\hline JetStrem G2 & $9(12,5 \%)$ & $4(3 \%)$ & 0.0137 \\
\hline CSI360 & $20(27,5 \%)$ & $46(34 \%)$ & 0.3489 \\
\hline Silver Hawk & $43(50 \%)$ & $82(62 \%)$ & 0.6549 \\
\hline
\end{tabular}

TASC — TransAtlantic Inter-Society Consensus

Table 3. Periprocedural complications.

\begin{tabular}{lccc}
\hline & Non-diabetic patients & Diabetic patients & $\mathbf{P}$ \\
\hline Artery perforation & $1(1.3 \%)$ & $0(0 \%)$ & 1.0 \\
Distal embolization & $0(0 \%)$ & $1(0.7 \%)$ & 1.0 \\
Flow limiting dissection & $2(2.7 \%)$ & $2(1.4 \%)$ & 1.0 \\
Bailout stenting & $2(2.7 \%)$ & $2(1.4 \%)$ & 1.0 \\
\hline
\end{tabular}

between the groups in the choice of atherectomy, except for JetStream in favor in the case of the nDM group (Table 2).

The number of periprocedural complications was similar between the groups. The detailed periprocedural outcomes are shown in Table 3.

At follow-up there were no differences between the groups in TLR after 6 months (DM: $7.5 \%$ vs. nDM $2.8 \%, \mathrm{p}=0.224), 12$ months (DM: $13.6 \%$ vs. $\mathrm{nDM} 20.8 \%, \mathrm{p}=0.232$ ) or 24 months (DM: $15.2 \%$ vs. $\mathrm{nDM} 22.2 \%, \mathrm{p}=0.249$ ) as shown in Figure 1. The amputation and death ratios were comparable between the groups (DM: $3 \%$ vs. $\mathrm{nDM} 1.5 \%$, $\mathrm{p}=\mathrm{NS}$ ) and (DM: $2.2 \%$ vs. $\mathrm{nDM} 2.7 \%, \mathrm{p}=\mathrm{NS}$ ), respectively, as also shown in Figure 1 . In the Kaplan-Mayer analysis, there were no differences in TLR-free survival, amputation free survival and survival $(\mathrm{p}=0.27$, hazard ratio $[\mathrm{HR}] 0.714,95 \%$ confidence interval [Cl] 0.371-1.314; $\mathrm{p}=0.81, \mathrm{HR}$ $0.8,95 \% \mathrm{Cl} 0.127-5.041 ; \mathrm{p}=0.557, \mathrm{HR} 4.542,95 \%$ 


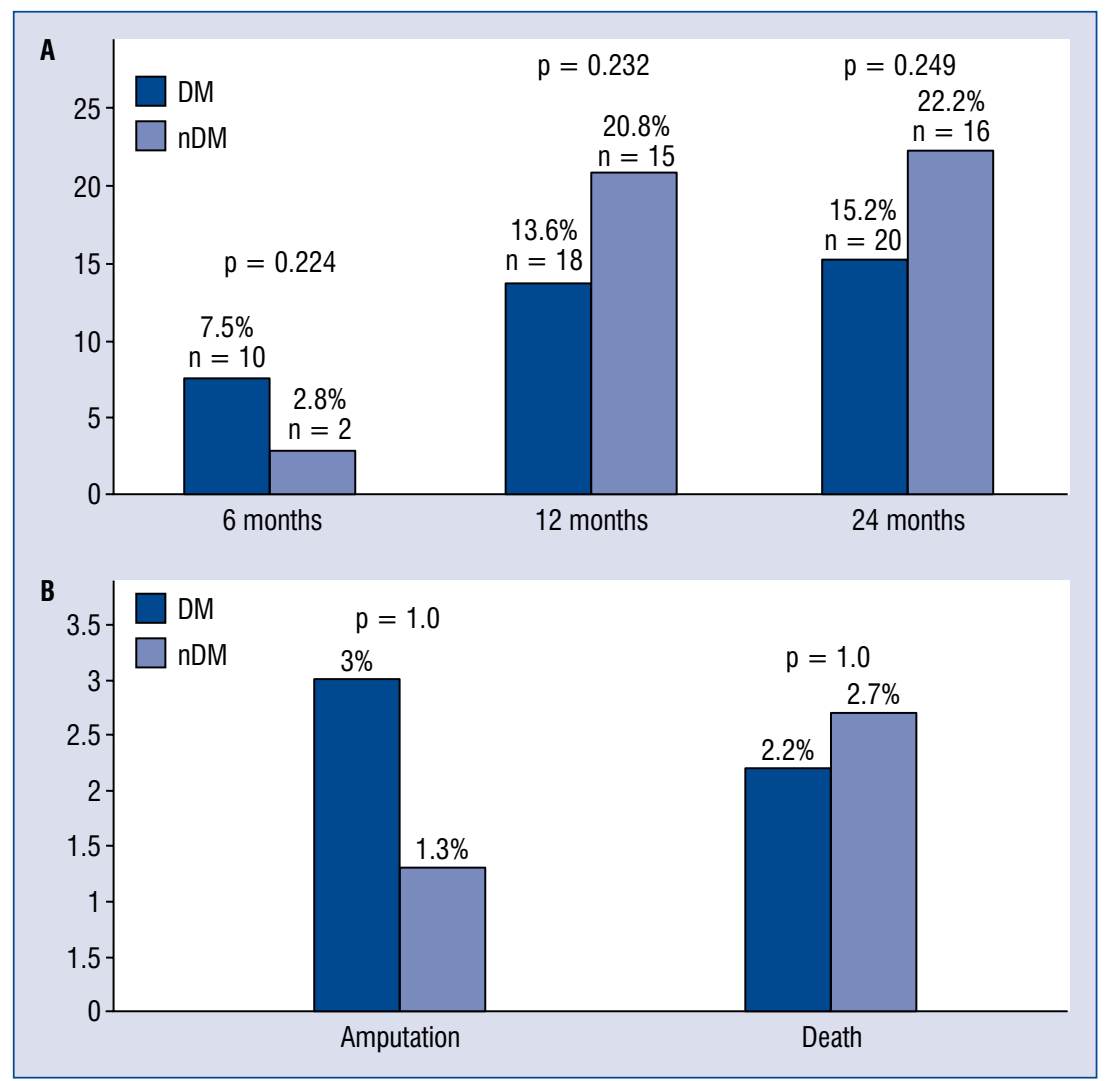

Figure 1. Target lesion revascularization (A), amputation and death (B) ratio; DM — diabetic patients; nDM - non-diabetic patients.

Cl 0.562-36.69), respectively, as shown in Figure 2. Moreover, there were no differences in the TLR between the groups depending on the artery and type of atherectomy device.

There were no significant differences in the Rutheford class between the groups during followup. However, there was a significant drop in the Rutherford class between groups before and after revascularization $(<0.0001)$ as shown in Figure 3.

\section{Discussion}

The current study presents a direct observational comparison of patients revascularized with atherectomy chosen based on plaque morphology in $\mathrm{DM}$ and $\mathrm{nDM}$ patients. According to available research, the present study, for the first-time, describes a direct comparison of long-term outcomes for different types atherectomies in diabetics vs. non-diabetics in claudicates as well as in critical limb ischemia patients. In this study, despite some discrepancies in patient baseline characteristics in favor of the nDM group, there were no differences in periprocedural complications, target lesion revascularization, amputation or death. It should be noted that the DM group consisted of high-risk patients for major cardiovascular adverse events due to numerous risk factors like end-stage renal disease, advanced coronary artery disease and obesity. Moreover, lesion characteristics are comparable between the groups. The difficulties treating PAD in diabetic patients have been driven by numerous factors including diffuse atherosclerosis causing longer lesions with smaller diameter lumen, more calcifications and greater plaque burden [10]. Furthermore, DM is associated with a more severe below-the-knee PAD, whereas risk factors, such as smoking, are associated with more proximal lesions [8].

There is very little data comparing long-term outcomes after treatment in patients with DM vs. $\mathrm{nDM}$ in PAD. A sub-analysis of Definitive Le comparing revascularization with SliverHawk/ /TurboHawk in diabetics and non-diabetics showed that directional atherectomy is equally effective in both groups of patients [11]. The ratio of target lesion revascularization was similar between the groups at 12-month follow-up and equaled $83.8 \%$ 


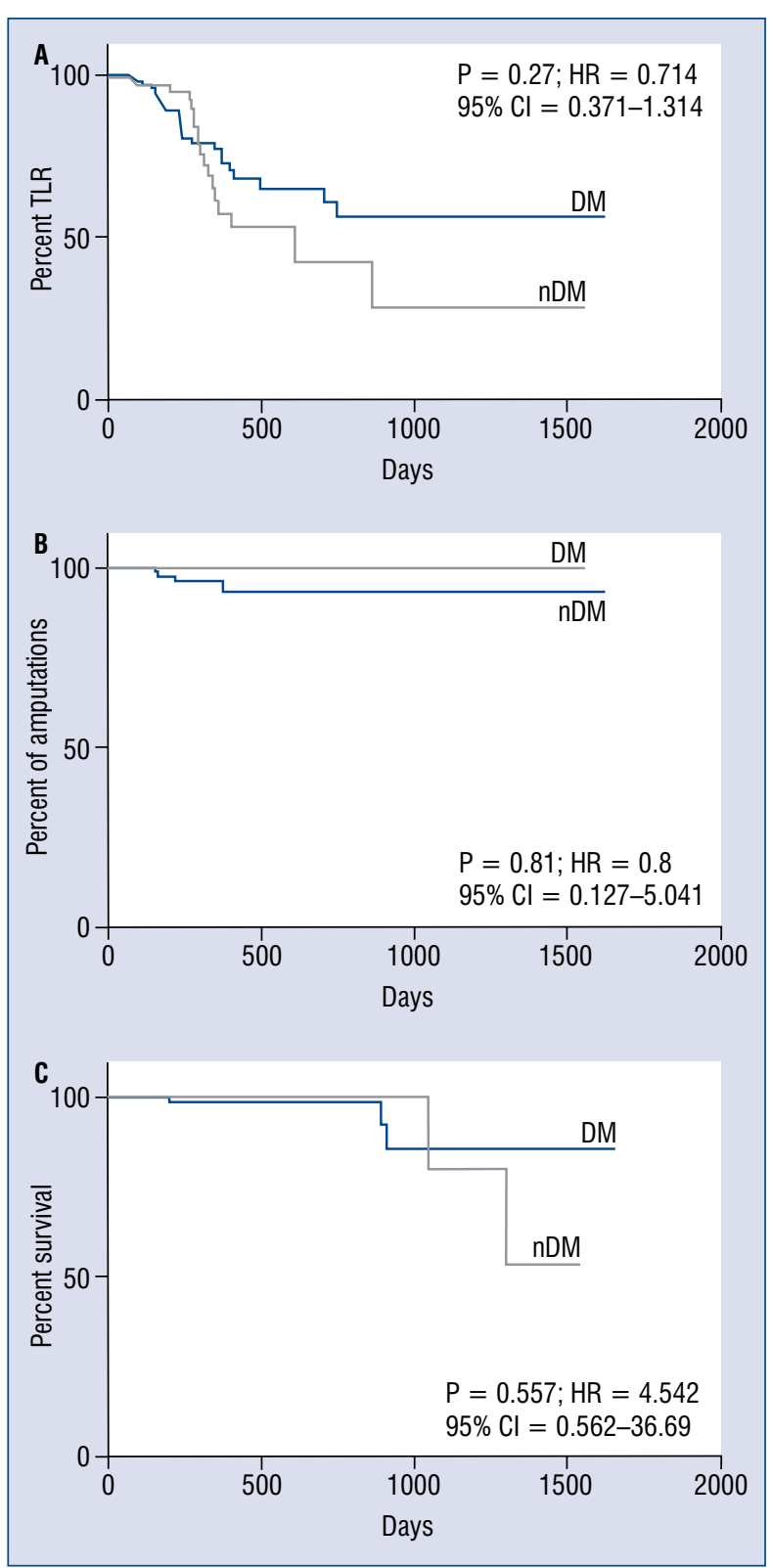

Figure 2. Kaplan-Mayer curves showing target lesion revascularization (TLR) free survival time $(\mathbf{A})$, amputation free survival time (B) and survival time (C); DM diabetic patients; nDM - non-diabetic patients.

and $87.5 \%$ for diabetics and non-diabetics, respectively. Just as in our database, the revascularization in Definitive Le was more frequent in case of below the knee procedures and the characteristics of demographics were similar. Nevertheless, in the Definitive Le study patients with critical limb ischemia were excluded. Lee et al. [6] compared the efficiency of plain old balloon angioplasty (POBA) in DM and nDM patients [6]. This study with a 2-year follow-up showed that POBA is less effective in diabetic patients, with a higher rate of restenosis and amputations. On the other hand, the drug eluting balloon (DEB) in the small study showed better outcomes in comparison to POBA in below-the-knee lesions at 3-month follow-up. Nevertheless, no benefits of DEB after 12 months were reported [12]. While comparing the stent technology, the Zilver PTX study reported that $\mathrm{DM}$ and $\mathrm{nDM}$ cohorts in their study had similar outcomes using the paclitaxel eluting stent [13]. Nonetheless, only superficial femoral artery was included as the target vessel. Darling et al. [7] published a direct comparison of diabetics and non-diabetics treated with POBA or bypass surgery in CLI patients. According to observations of this group, diabetics manifested an increased risk of long-term mortality, incomplete wound healing, a major amputation and restenosis, especially after POBA in comparison to non-diabetics. Furthermore, Dick et al. [14] published a study with results similar to the study mentioned earlier.

There is an interesting technology that may by combined with atherectomy in PAD and it is the local drug delivery after revascularization. Early reports on the combination of plaque modification with atherectomy and subsequent DEB seem to be promising $[15,16]$. Novel technologies, including local drug delivery nano-technology, may soon become available for follow-up treatment of plaque modifications after atherectomy [17].

All patients in this study were also treated pharmacologically to reduce any major cardiovascular adverse events. Despite encouraging data on including ciliostazol in the treatment after stenting of femoropopliteal region [18], almost all the present patients were on dual antiplatelet therapy consisting of clopidogrel (75 mg) and acetylsalicylic acid (81 mg) once a day. Dual antiplatelet therapy was prescribed due to the fact that after AT, the intima-media could be exposed to blood flow, significantly increasing the risk of acute or subacute thrombosis [19].

To summarize, this study shows that the outcomes of atherectomy in PAD are similar in DM patients as compared to $\mathrm{nDM}$ patients. The large minimal lumen diameter obtained during atherectomy may play a crucial role in this phenomenon, which translates into a lower TLR ratio at follow-up in diabetics as well as non-diabetics.

\section{Limitations of the study}

The main drawbacks of this analysis are those inherent to any single-center, observational study [20], along with differences in baseline patient 


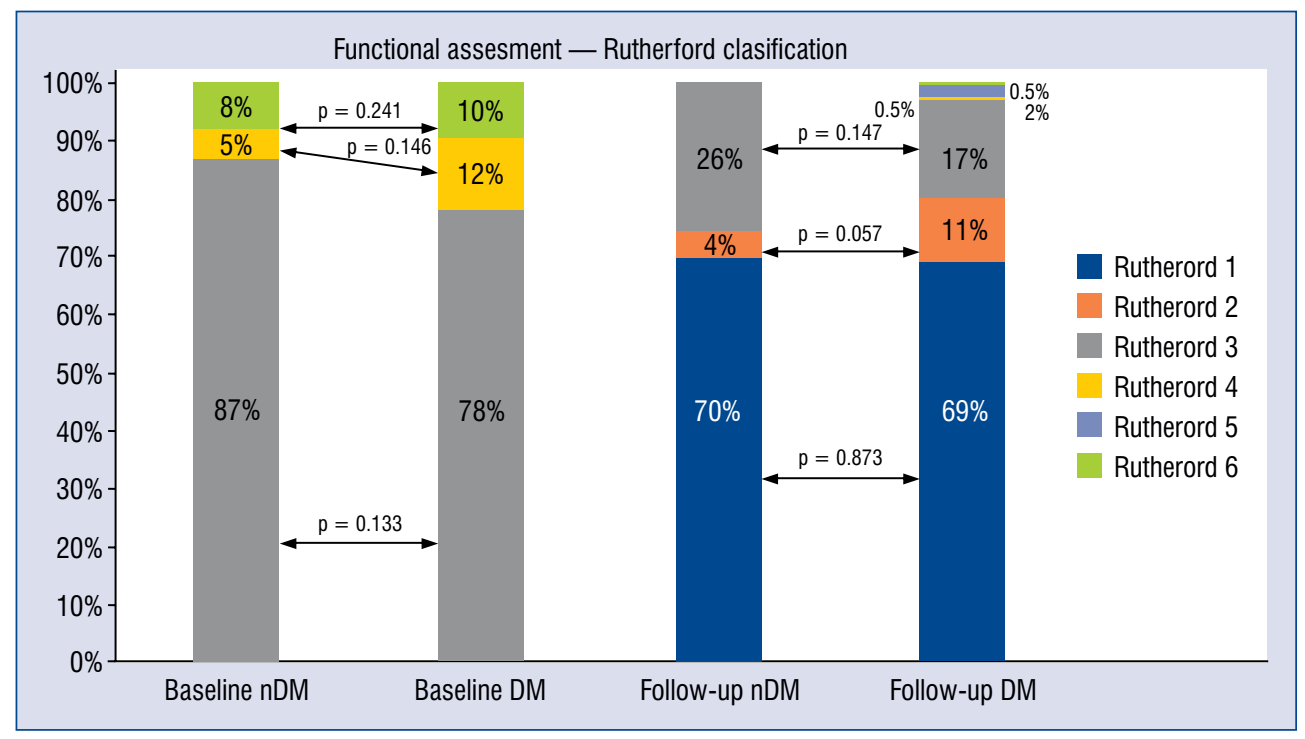

Figure 3. Rutherford classification prior and after treatment. DM - diabetic patients; nDM — non-diabetic patients.

characteristics. Nevertheless, the differences arise from the character of DM and nDM patients. The exact data on very long below the knee chronic total occlusion are unavailable. The ankle brachial index, ultrasonography with Doppler and toe pressure were not performed on each visit, making this data unsuitable for statistical analysis. This study is hypothesis-generating only.

\section{Conclusions}

In this hypothesis-generating study of patients with lower extremity PAD, plaque modifications with adjusted atherectomies appear to have similar outcomes in diabetic as well as in non-diabetic patients. Nevertheless, this should be confirmed in further controlled randomized trials.

\section{Conflict of interest: None declared}

\section{References}

1. Whiting DR, Guariguata L, Weil C, et al. IDF diabetes atlas: global estimates of the prevalence of diabetes for 2011 and 2030. Diabetes Res Clin Pract. 2011; 94(3): 311-321, doi: 10.1016/j. diabres.2011.10.029, indexed in Pubmed: 22079683.

2. Fowkes FG, Rudan D, Rudan I, et al. Comparison of global estimates of prevalence and risk factors for peripheral artery disease in 2000 and 2010: a systematic review and analysis. Lancet. 2013; 382(9901): 1329-1340, doi: 10.1016/S0140-6736(13)61249-0, indexed in Pubmed: 23915883.

3. Criqui MH. Peripheral arterial disease--epidemiological aspects. Vasc Med. 2001; 6(3 Suppl): 3-7, doi: 10.1177/1358836X010060 0i102, indexed in Pubmed: 11789963.
4. O'Hare AM, Glidden DV, Fox CS, et al. High prevalence of peripheral arterial disease in persons with renal insufficiency: results from the National Health and Nutrition Examination Survey 1999-2000. Circulation. 2004; 109(3): 320-323, doi: 10.1161/01. CIR.0000114519.75433.DD, indexed in Pubmed: 14732743.

5. Suzuki JI, Shimamura M, Suda H, et al. Current therapies and investigational drugs for peripheral arterial disease. Hypertens Res. 2016; 39(4): 183-191, doi: 10.1038/hr.2015.134, indexed in Pubmed: 26631852.

6. Lee MS, Rha SW, Han SK, et al. Comparison of diabetic and non-diabetic patients undergoing endovascular revascularization for peripheral arterial disease. J Invasive Cardiol. 2015; 27(3): 167-171, indexed in Pubmed: 25740971.

7. Darling JD, Bodewes TCF, Deery SE, et al. Outcomes after first-time lower extremity revascularization for chronic limbthreatening ischemia between patients with and without diabetes. J Vasc Surg. 2018; 67(4): 1159-1169, doi: 10.1016/j. jvs.2017.06.119, indexed in Pubmed: 28947228.

8. Jude EB, Oyibo SO, Chalmers N, et al. Peripheral arterial disease in diabetic and nondiabetic patients: a comparison of severity and outcome. Diabetes Care. 2001; 24(8): 1433-1437, indexed in Pubmed: 11473082.

9. Janas A, Buszman PP, Milewski KP, et al. Long-Term outcomes after percutaneous lower extremity arterial interventions with atherectomy vs. Balloon angioplasty: propensity score-matched registry. Circ J. 2017; 81(3): 376-382, doi: 10.1253/circj.CJ-160856, indexed in Pubmed: 28090010.

10. Marso SP, Hiatt WR. Peripheral arterial disease in patients with diabetes. J Am Coll Cardiol. 2006; 47(5): 921-929, doi: 10.1016/j. jacc.2005.09.065, indexed in Pubmed: 16516072.

11. Garcia LA, Jaff MR, Rocha-Singh KJ, et al. A comparison of clinical outcomes for diabetic and nondiabetic patients following directional atherectomy in the DEFINITIVE LE claudicant cohort. J Endovasc Ther. 2015; 22(5): 701-711, doi: 10.1177/1526602815599550, indexed in Pubmed: 26250748.

12. Oz II, Serifoglu I, Bilici M, et al. Comparison of drug-eluting balloon and standard balloon angioplasty for infrapopliteal ar- 
terial diseases in diabetic patients. Vasc Endovascular Surg. 2016; 50(8): 534-540, doi: 10.1177/1538574416676019, indexed in Pubmed: 28081691.

13. Fanelli F, Di Primo M, Boatta E, et al. Drug-eluting nitinol stent treatment of the superficial femoral artery and above-the-knee popliteal artery (the Zilver PTX single-arm clinical study): a comparison between diabetic and nondiabetic patients. Cardiovasc Intervent Radiol. 2013; 36(5): 1232-1240, doi: 10.1007/ /s00270-012-0543-5, indexed in Pubmed: 23299704.

14. Dick F, Diehm N, Galimanis A, et al. Surgical or endovascular revascularization in patients with critical limb ischemia: influence of diabetes mellitus on clinical outcome. J Vasc Surg. 2007; 45(4): 751-761, doi: 10.1016/j.jvs.2006.12.022, indexed in Pubmed: 17306950.

15. Stavroulakis K, Bisdas T, Torsello G, et al. Combined directional atherectomy and drug-eluting balloon angioplasty for isolated popliteal artery lesions in patients with peripheral artery disease. J Endovasc Ther. 2015; 22(6): 847-852, doi: 10.1177/1526602815608194, indexed in Pubmed: 26420802.

16. Tellez A, Dattilo R, Mustapha JA, et al. Biological effect of orbital atherectomy and adjunctive paclitaxel-coated balloon ther- apy on vascular healing and drug retention: early experimental insights into the familial hypercholesterolaemic swine model of femoral artery stenosis. EuroIntervention. 2014; 10(8): 1002 -1008, doi: 10.4244/EIJY14M10_03, indexed in Pubmed: 25308298.

17. Granada JF, Tellez A, Baumbach WR, et al. In vivo delivery and long-term tissue retention of nano-encapsulated sirolimus using a novel porous balloon angioplasty system. EuroIntervention. 2016; 12(6): 740-747, doi: 10.4244/EIJY15M10_01, indexed in Pubmed: 26428893.

18. Iida $\mathrm{O}$, Yokoi $\mathrm{H}$, Soga $\mathrm{Y}$, et al. Cilostazol reduces angiographic restenosis after endovascular therapy for femoropopliteal lesions in the Sufficient Treatment of Peripheral Intervention by Cilostazol study. Circulation. 2013; 127(23): 2307-2315, doi: 10.1161/CIRCULATIONAHA.112.000711, indexed in Pubmed: 23652861.

19. Andrews RK, Berndt MC. Platelet physiology and thrombosis. Thromb Res. 2004; 114(5-6): 447-453, doi: 10.1016/j. thromres.2004.07.020, indexed in Pubmed: 15507277.

20. Biondi-Zoccai GG, Agostoni P, Abbate A. Parallel hierarchy of scientific studies in cardiovascular medicine. Ital Heart J. 2003; 4(11): 819-820, indexed in Pubmed: 14699715. 